PROCEEDINGS OF THE

AMERICAN MATHEMATICAL SOCIETY

Volume 138, Number 1, January 2010, Pages 199-207

S 0002-9939(09)10049-7

Article electronically published on August 19, 2009

\title{
A NOTE ON DECAY RATES FOR SCHRÖDINGER'S EQUATION
}

\author{
JIAN XIE, LINZI ZHANG, AND THIERRY CAZENAVE
}

(Communicated by Walter Craig)

\begin{abstract}
We prove the existence of solutions of the Schrödinger equation on $\mathbb{R}^{N}$ which decay, in various $L^{p}$ spaces, at different rates along different time sequences going to infinity. We establish a similar result for a nonlinear Schrödinger equation.
\end{abstract}

\section{INTRODUCTION}

In this paper, we study the decay rates as $t \rightarrow \infty$, in various $L^{p}$ spaces, of the solution of Schrödinger's equation in $\mathbb{R}^{N}$ :

$$
\left\{\begin{array}{l}
i u_{t}+\Delta u=0 \\
u(0)=\varphi
\end{array}\right.
$$

As is well-known, given any $\varphi \in \mathcal{S}^{\prime}\left(\mathbb{R}^{N}\right)$, the solution of (1.1) is

$$
u(t)=e^{i t \Delta} \varphi=K_{t} \star \varphi,
$$

where the kernel $K_{t}$ is given by

$$
K_{t}(x)=(4 \pi i t)^{-\frac{N}{2}} e^{\frac{i|x|^{2}}{4 t}} .
$$

It follows from (1.2)-(1.3) that if $\varphi \in L^{1}\left(\mathbb{R}^{N}\right)$, then $|t|^{\frac{N}{2}}\|u(t)\|_{L^{\infty}} \leq\|\varphi\|_{L^{1}}$. In addition, the Fourier transform of formula (1.2) implies that $e^{i t \Delta}$ is an isometry of $L^{2}\left(\mathbb{R}^{N}\right)$. The Riesz-Thorin interpolation theorem then shows that if $2 \leq q \leq \infty$ and $\varphi \in L^{q^{\prime}}\left(\mathbb{R}^{N}\right)$, then

$$
|t|^{\frac{N}{2}\left(1-\frac{2}{q}\right)}\|u(t)\|_{L^{q}} \leq\|\varphi\|_{L^{q^{\prime}}} .
$$

The power of $|t|$ in (1.4) is known to be optimal. (The constant 1 is not optimal; see [1.) Indeed, given any $\varphi \in \mathcal{S}^{\prime}\left(\mathbb{R}^{N}\right), \varphi \neq 0$ and $2 \leq q \leq \infty$, it follows that

$$
\liminf _{t \rightarrow \pm \infty}|t|^{\frac{N}{2}\left(1-\frac{2}{q}\right)}\|u(t)\|_{L^{q}}>0 .
$$

(See e.g.: Lemma, p. 62 in [12; Decay Lemma, p. 228 in [11; and Proposition 8.1 in 7 for three different proofs.) On the other hand, some solutions decay more slowly than the fastest decay rate. Indeed, given any $2<q \leq \infty$, if $0<\nu<\frac{N}{2}\left(1-\frac{2}{q}\right)$ and $\varphi(x)=|x|^{-2 \nu-\frac{N}{q}}$, then $\|u(t)\|_{L^{q}}=c|t|^{-\nu}$ for some $c>0$. (See Remark 8.2

Received by the editors March 3, 2009.

2000 Mathematics Subject Classification. Primary 35Q55.

Key words and phrases. Schrödinger's equation, asymptotic behavior, decay rate.

The first two authors were supported by NSFC 10871175.

(C)2009 American Mathematical Society Reverts to public domain 28 years from publication 
in [7.) Thus we see that any decay rate slower than the maximal one is achieved by some solutions. However, certain solutions may have no definite decay rate, as shown in the following theorem.

Theorem 1.1. Given any $2 \leq r \leq \infty$, there exists a real valued, positive, radially symmetric and decreasing function $\varphi \in W^{m, r}\left(\mathbb{R}^{N}\right) \cap W^{m, \infty}\left(\mathbb{R}^{N}\right)$ for all $m \geq 0$ with the following properties:

(i) The mapping $t \mapsto e^{i t \Delta} \varphi$ is continuous $\mathbb{R} \rightarrow W^{m, r}\left(\mathbb{R}^{N}\right) \cap W^{m, \infty}\left(\mathbb{R}^{N}\right)$ for all $m \geq 0$.

(ii) For all $r<q \leq \infty, \sup _{t \geq 0}(1+t)^{\frac{N}{2}\left(\frac{1}{r}-\frac{1}{q}\right)}\left\|e^{i t \Delta} \varphi\right\|_{L^{q}}<\infty$.

(iii) For all $2<q \leq \infty, q \geq r$, all

$$
N>\sigma>\frac{N}{2}\left(\frac{1}{r}-\frac{1}{q}\right)\left(\frac{1}{2}-\frac{1}{q}\right)^{-1}
$$

and all $0 \leq c<\infty$, there exists a sequence $t_{n} \rightarrow \infty$ such that

$$
t_{n}^{\frac{\sigma}{2}\left(1-\frac{2}{q}\right)}\left\|e^{i t_{n} \Delta} \varphi\right\|_{L^{q}} \rightarrow c
$$

as $n \rightarrow \infty$.

Note that the initial value $\varphi$ is real-valued, so that $e^{-i t \Delta} \varphi=\overline{e^{i t \Delta} \varphi}$ for all $t \in \mathbb{R}$. Thus the properties stated in parts (ii) and (iii) for positive times have counterparts for negative times.

Given any $r<q \leq \infty$, the solution $e^{i t \Delta} \varphi$ of Theorem 1.1 decays in $L^{q}\left(\mathbb{R}^{N}\right)$ at least as does $t^{-\frac{N}{2}\left(\frac{1}{r}-\frac{1}{q}\right)}$ by part (ii). It decays at most as does $t^{-N\left(\frac{1}{2}-\frac{1}{q}\right)}$ by $(1.5)$. On the other hand, it achieves all the decay rates in between $t^{-N\left(\frac{1}{2}-\frac{1}{q}\right)}$ and $t^{-\frac{N}{2}\left(\frac{1}{r}-\frac{1}{q}\right)}$ along some sequence $t_{n} \rightarrow \infty$. Indeed, given any $c>0$ and $\frac{N}{2}\left(\frac{1}{r}-\frac{1}{q}\right)<\nu<$ $N\left(\frac{1}{2}-\frac{1}{q}\right)$, it follows from part (iii) that it achieves the rate $c t^{-\nu}$ along some sequence $t_{n} \rightarrow \infty$.

Finite energy solutions of (1.1) being of particular relevance, we emphasize in the following corollary the case $r=2$, in which the initial value belongs to $H^{\infty}\left(\mathbb{R}^{N}\right)$.

Corollary 1.2. There exists a real valued, positive, radially symmetric and decreasing function $\varphi \in H^{m}\left(\mathbb{R}^{N}\right) \cap W^{m, \infty}\left(\mathbb{R}^{N}\right)$ for all $m \geq 0$ with the following properties:

(i) The mapping $t \mapsto e^{i t \Delta} \varphi$ is continuous $\mathbb{R} \rightarrow H^{m}\left(\mathbb{R}^{N}\right) \cap W^{m, \infty}\left(\mathbb{R}^{N}\right)$ for all $m \geq 0$.

(ii) For all $2<q \leq \infty$, $\sup _{t \geq 0}(1+t)^{\frac{N}{2}\left(\frac{1}{2}-\frac{1}{q}\right)}\left\|e^{i t \Delta} \varphi\right\|_{L^{q}}<\infty$.

(iii) For all $2<q \leq \infty$, all $N>\sigma>N / 2$ and all $0 \leq c<\infty$, there exists a sequence $t_{n} \rightarrow \infty$ such that $t_{n}^{\sigma\left(\frac{1}{2}-\frac{2}{q}\right)}\left\|e^{i t_{n} \Delta} \varphi\right\|_{L^{q}} \rightarrow$ c as $n \rightarrow \infty$.

Letting $q=r>2$ in part (iii) of Theorem 1.1, the condition (1.6) becomes $0<\sigma<N$, and we obtain the following corollary, in which the solution $e^{i t \Delta} \varphi$ of Theorem 1.1 achieves all the decay rates slower than the maximal rate $t^{-N\left(\frac{1}{2}-\frac{1}{q}\right)}$.

Corollary 1.3. Given any $2<r \leq \infty$, there exists a real valued, positive, radially symmetric and decreasing function $\varphi \in W^{m, r}\left(\mathbb{R}^{N}\right) \cap W^{m, \infty}\left(\mathbb{R}^{N}\right)$ for all $m \geq 0$ with the following properties:

(i) The mapping $t \mapsto e^{i t \Delta} \varphi$ is continuous $\mathbb{R} \rightarrow W^{m, r}\left(\mathbb{R}^{N}\right) \cap W^{m, \infty}\left(\mathbb{R}^{N}\right)$ for all $m \geq 0$. 
(ii) For all $0<\sigma<N$ and all $0 \leq c<\infty$, there exists a sequence $t_{n} \rightarrow \infty$ such that $t_{n}^{\sigma\left(\frac{1}{2}-\frac{1}{r}\right)}\left\|e^{i t_{n} \Delta} \varphi\right\|_{L^{r}} \rightarrow c$ as $n \rightarrow \infty$.

Remark 1.4. In [8, it is shown that, given any $0<\sigma<N$, there exists an initial value $\varphi$ for which the set of limits in $L^{q}\left(\mathbb{R}^{N}\right)$, along sequences, of $t^{\frac{\sigma}{2}}\left[e^{i t \Delta} \varphi\right](\cdot \sqrt{t})$ is "large" in the sense that, for all sufficiently large $q$, it is a closed ball of an infinite dimensional Banach space. In view of Theorem 1.1 and Corollary 1.3. one may wonder if, for a given initial value $\varphi$, this set can be large for various values of the scaling parameter $\sigma$. This is indeed the case. In the article 9 , the authors construct initial values for which this set is the whole space $L^{q}\left(\mathbb{R}^{N}\right)$ for an infinite set $\left(\sigma_{n}\right)_{n \geq 1} \subset(0, N)$ of scaling parameters.

We now consider the nonlinear Schrödinger equation in $\mathbb{R}^{N}$ :

$$
\left\{\begin{array}{l}
i u_{t}+\Delta u=\lambda|u|^{\alpha} u \\
u(0)=\varphi
\end{array}\right.
$$

where $\lambda \in \mathbb{R}$,

$$
\frac{4}{N}<\alpha<\frac{4}{(N-2)^{+}},
$$

and $\varphi \in H^{1}\left(\mathbb{R}^{N}\right)$ is a given initial value. It is well-known that if either $\lambda>0$ or $\lambda<0$ and $\|\varphi\|_{H^{1}}$ is sufficiently small, then there exists a unique, global and bounded solution of (1.8), i.e. $u \in C\left([0, \infty), H^{1}\left(\mathbb{R}^{N}\right)\right) \cap L^{\infty}\left((0, \infty), H^{1}\left(\mathbb{R}^{N}\right)\right)$. (See e.g. [10] for the case $\lambda>0$ and Theorem 6.2.1 in [3] for the case $\lambda<0$.) Assuming, in addition, $\varphi \in L^{2}\left(\mathbb{R}^{N},|x|^{2} d x\right)$, the solution $u$ satisfies

$$
\sup _{t>0} t^{N\left(\frac{1}{2}-\frac{1}{q}\right)}\|u(t)\|_{L^{q}}<\infty,
$$

for all $2<q<2 N /(N-2)^{+}$. (See Theorem 7.3.1 in 3] for the case $\lambda>0$ and Theorem 4.2 in $[5$ for the case $\lambda<0$.) Moreover, the decay rate (1.10) is optimal. Indeed, if $u$ is any global solution of (1.8) which is bounded in $H^{1}\left(\mathbb{R}^{N}\right)$, then

$$
\liminf _{t \rightarrow \infty} t^{N\left(\frac{1}{2}-\frac{1}{q}\right)}\|u(t)\|_{L^{q}}>0
$$

for all $2<q \leq \infty$. (See [2].) The following result shows that for the nonlinear equation (1.8) some solutions have multiple decay rates.

Theorem 1.5. Let $\lambda \in \mathbb{R}$ and assume (1.9). It follows that there exists a realvalued, positive, radially symmetric and decreasing function $\varphi \in H^{1}\left(\mathbb{R}^{N}\right)$ such that the corresponding solution $u$ of (1.8) satisfies the following properties:

(i) The solution $u$ is global and bounded in $H^{1}\left(\mathbb{R}^{N}\right)$, uniformly in $t \geq 0$.

(ii) There exists $\alpha+2<q_{0}<2 N /(N-2)^{+}$such that

$$
\sup _{t \geq 0}(1+t)^{\frac{N}{2}\left(\frac{1}{2}-\frac{1}{q}\right)}\|u(t)\|_{L^{q}}<\infty,
$$

for all $2<q<q_{0}$. Moreover, given any $2<q<q_{0}$, there exists $\delta(q)>0$ such that for all $0<\eta<\delta(q)$ and all $c>0$ there exists a sequence $t_{n} \rightarrow \infty$ such that

$$
t_{n}^{\frac{N}{2}\left(\frac{1}{2}-\frac{1}{q}\right)+\eta}\left\|u\left(t_{n}\right)\right\|_{L^{q}} \rightarrow c,
$$

as $n \rightarrow \infty$. 
We see that the solution $u$ given by Theorem 1.5 achieves, for every $2<q<q_{0}$, all the decay rates in $L^{q}\left(\mathbb{R}^{N}\right)$ between $t^{-\frac{N}{2}\left(\frac{1}{2}-\frac{1}{q}\right)}$ and $t^{-\frac{N}{2}\left(\frac{1}{2}-\frac{1}{q}\right)-\delta(q)}$ along some sequence $t_{n} \rightarrow \infty$.

\section{Proofs of Theorems 1.1 And 1.5}

Theorem 1.1]is proved by explicit constructions, inspired by [4, where a result of a similar nature is established for the heat equation. The argument in [4] uses the property that $e^{t \Delta}$ is a contraction on $L^{\infty}$. The Schrödinger group does not satisfy that property, so instead we use the following elementary estimate.

Lemma 2.1. If

$$
\theta(x)=e^{-|x|^{2}}
$$

then

$$
\left\|e^{i t \Delta}[\theta(\gamma \cdot)]\right\|_{L^{q}}=\left(1+16 t^{2} \gamma^{4}\right)^{-\frac{N}{2}\left(\frac{1}{2}-\frac{1}{q}\right)} \gamma^{-\frac{N}{q}}\left(\frac{\pi}{q}\right)^{\frac{N}{2 q}},
$$

for all $t \in \mathbb{R}, \gamma>0$ and $1 \leq q \leq \infty$, with the convention that $(\pi / q)^{\frac{N}{2 q}}=1$ if $q=\infty$.

Proof. It follows from an easy calculation that

$$
e^{i t \Delta}[\theta(\gamma \cdot)]=\left(1+4 i t \gamma^{2}\right)^{-\frac{N}{2}} e^{-\frac{\gamma^{2}|x|^{2}}{1+4 i t \gamma^{2}}}
$$

so that

$$
\left|e^{i t \Delta}[\theta(\gamma \cdot)]\right|=\left(1+16 t^{2} \gamma^{4}\right)^{-\frac{N}{4}} e^{-\frac{\gamma^{2}|x|^{2}}{1+16 t^{2} \gamma^{4}}}
$$

Therefore,

$$
\left\|e^{i t \Delta}[\theta(\gamma \cdot)]\right\|_{L^{q}}=\left(1+16 t^{2} \gamma^{4}\right)^{-\frac{N}{2}\left(\frac{1}{2}-\frac{1}{q}\right)} \gamma^{-\frac{N}{q}}\|\theta\|_{L^{q}}
$$

and (2.2) follows.

Proof of Theorem 1.1. We give the proof in the case where both the exponents $r$ and $q$ are finite. It is not difficult to see that the argument applies with obvious interpretations if either exponent is infinite. Let $\theta$ be defined by (2.1), let the sequence $\left(a_{j}\right)_{j \geq 0}$ be defined by

$$
\left\{\begin{array}{l}
a_{1}=a_{0}=1, \\
a_{j+1}=\exp \left(\exp a_{j}\right) \quad j \geq 1,
\end{array}\right.
$$

and set

$$
\varphi(x)=\sum_{j=1}^{\infty} a_{j}^{-\frac{N}{r}} e^{-2 a_{j-1}} \theta\left(\frac{x}{a_{j}}\right) .
$$

We first show property (i). Since $\theta \in \mathcal{S}\left(\mathbb{R}^{N}\right)$, the function $t \mapsto e^{i t \Delta}\left[\theta\left(\cdot / a_{j}\right)\right]$ is continuous $[0, \infty) \rightarrow L^{r}\left(\mathbb{R}^{N}\right) \cap C_{0}\left(\mathbb{R}^{N}\right)$ for every $j \geq 1$. Therefore, to prove property (i) for $m=0$, we need only show that

$$
\sum_{j=n}^{\infty} a_{j}^{-\frac{N}{r}} e^{-2 a_{j-1}}\left(\left\|e^{i t \Delta}\left[\theta\left(\cdot / a_{j}\right)\right]\right\|_{L^{r}}+\left\|e^{i t \Delta}\left[\theta\left(\cdot / a_{j}\right)\right]\right\|_{L^{\infty}}\right) \underset{n \rightarrow \infty}{\longrightarrow} 0,
$$

uniformly for $t$ in a bounded subset of $[0, \infty)$. We deduce from (2.2) that there exists a constant $C$ such that

$$
\left\|e^{i t \Delta}\left[\theta\left(\cdot / a_{j}\right)\right]\right\|_{L^{r}}+\left\|e^{i t \Delta}\left[\theta\left(\cdot / a_{j}\right)\right]\right\|_{L^{\infty}} \leq C a_{j}^{\frac{N}{r}},
$$


and (2.5) easily follows using (2.3). To prove property (i) for $m \geq 1$, we observe that every derivative applied to $\varphi$ produces a factor $a_{j}^{-1} \leq 1$ in each term of the sum (2.4). Therefore, the result follows immediately from the above calculations.

We next prove property (ii). Given $r<q \leq \infty$, we have

$$
\left\|e^{i t \Delta} \varphi\right\|_{L^{q}} \leq \sum_{j=1}^{\infty} a_{j}^{-\frac{N}{r}} e^{-2 a_{j-1}}\left\|e^{i t \Delta}\left[\theta\left(\cdot / a_{j}\right)\right]\right\|_{L^{q}} .
$$

Fix $n \geq 1$. Since $\left\|e^{i t \Delta}\left[\theta\left(\cdot / a_{j}\right)\right]\right\|_{L^{q}} \leq C a_{j}^{\frac{N}{q}}$ by $(\underline{2.2})$, we see that

$$
\begin{aligned}
& \sum_{j=n+1}^{\infty} a_{j}^{-\frac{N}{r}} e^{-2 a_{j-1}}\left\|e^{i t \Delta}\left[\theta\left(\cdot / a_{j}\right)\right]\right\|_{L^{q}} \\
& \quad \leq C \sum_{j=n+1}^{\infty} a_{j}^{-\frac{N(q-r)}{q r}} e^{-2 a_{j-1}} \leq C a_{n+1}^{-\frac{N(q-r)}{q r}} \sum_{j=1}^{\infty} e^{-2 a_{j-1}} \leq C a_{n+1}^{-\frac{N(q-r)}{q r}} .
\end{aligned}
$$

Next, we deduce from $(2.2)$ that $\left\|e^{i t \Delta}\left[\theta\left(\cdot / a_{j}\right)\right]\right\|_{L^{q}} \leq C a_{j}^{\frac{N(q-1)}{q}} t^{-\frac{N(q-2)}{2 q}}$, and so

$$
\begin{array}{r}
\sum_{j=1}^{n} a_{j}^{-\frac{N}{r}} e^{-2 a_{j-1}}\left\|e^{i t \Delta}\left[\theta\left(\cdot / a_{j}\right)\right]\right\|_{L^{q}} \leq C t^{-\frac{N(q-2)}{2 q}} \sum_{j=1}^{n} a_{j}^{\frac{N[q(r-1)-r]}{q r}} e^{-2 a_{j-1}} \\
\leq C t^{-\frac{N(q-2)}{2 q}} a_{n}^{\frac{N[q(r-1)-r]}{q r}} \sum_{j=1}^{\infty} e^{-2 a_{j-1}} \leq C t^{-\frac{N(q-2)}{2 q}} a_{n}^{\frac{N[q(r-1)-r]}{q r}}
\end{array}
$$

We deduce from (2.6), (2.7) and (2.8) that

$$
\left\|e^{i t \Delta} \varphi\right\|_{L^{q}} \leq C\left(t^{-\frac{N(q-2)}{2 q}} a_{n}^{\frac{N[q(r-1)-r]}{q r}}+a_{n+1}^{-\frac{N(q-r)}{q r}}\right) .
$$

Given $t \geq 1$, we consider the unique value $n=n(t)$ such that

$$
a_{n}^{2} \leq t<a_{n+1}^{2} .
$$

It follows from (2.9) and (2.10) that $\left\|e^{i t \Delta} \varphi\right\|_{L^{q}} \leq C t^{-\frac{N(q-r)}{2 q r}}$. Since $\| e^{i t \Delta} \varphi_{L^{q}}$ is bounded for $0 \leq t \leq 1$ by property (i), this proves the desired estimate.

We now prove property (iii). We fix $c>0,2<q \leq \infty, q \geq r, \sigma$ satisfying (1.6), and we set

$$
\lambda_{n}=\left(2^{-N} c^{-\frac{q}{q-2}}\left(\frac{\pi}{q}\right)^{\frac{N}{2(q-2)}} e^{-\frac{2 q}{q-2} a_{n-1}} a_{n}^{N-\frac{N(q-r)}{r(q-2)}}\right)^{\frac{1}{N-\sigma}},
$$

for all $n \geq 1$, so that $\lambda_{n} \rightarrow \infty$ as $n \rightarrow \infty$, by (2.3) and (1.6). We write

$$
e^{i \lambda_{n}^{2} \Delta} \varphi=U_{n}+V_{n}+W_{n}
$$

where

$$
\begin{aligned}
U_{n} & =\sum_{j=1}^{n-1} a_{j}^{-\frac{N}{r}} e^{-2 a_{j-1}} e^{i \lambda_{n}^{2} \Delta}\left[\theta\left(\cdot / a_{j}\right)\right], \\
V_{n} & =a_{n}^{-\frac{N}{r}} e^{-2 a_{n-1}} e^{i \lambda_{n}^{2} \Delta}\left[\theta\left(\cdot / a_{n}\right)\right], \\
W_{n} & =\sum_{j=n+1}^{\infty} a_{j}^{-\frac{N}{r}} e^{-2 a_{j-1}} e^{i \lambda_{n}^{2} \Delta}\left[\theta\left(\cdot / a_{j}\right)\right] .
\end{aligned}
$$


It follows from (2.2) that

$$
\left\|e^{i \lambda_{n}^{2} \Delta}\left[\theta\left(\cdot / a_{j}\right)\right]\right\|_{L^{q}} \leq C\left(\lambda_{n} a_{j}^{-1}\right)^{-\frac{N(q-2)}{q}} a_{j}^{\frac{N}{q}} .
$$

Therefore, if $j \leq n-1$, then

$$
\begin{aligned}
a_{j}^{-\frac{N}{r}} e^{-2 a_{j-1}}\left\|e^{i \lambda_{n}^{2} \Delta}\left[\theta\left(\cdot / a_{j}\right)\right]\right\|_{L^{q}} & \leq C\left(\lambda_{n} a_{j}^{-1}\right)^{-\frac{N(q-2)}{q}} a_{j}^{\frac{N}{q}} a_{j}^{-\frac{N}{r}} e^{-2 a_{j-1}} \\
& \leq C \lambda_{n}^{-\frac{N(q-2)}{q}} a_{j}^{\frac{N(q-2)}{q}} \leq C \lambda_{n}^{-\frac{N(q-2)}{q}} a_{n-1}^{\frac{N(q-2)}{q}} .
\end{aligned}
$$

We deduce from the above inequality, (2.11) and (2.3) that

$$
\lambda_{n}^{\frac{\sigma(q-2)}{q}}\left\|U_{n}\right\|_{L^{q}} \leq C n \lambda_{n}^{-\frac{(N-\sigma)(q-2)}{q}} a_{n-1}^{\frac{N(q-2)}{q}} \underset{n \rightarrow \infty}{\longrightarrow} 0 .
$$

Next, it follows from (2.2) and (2.11) that

$$
\lambda_{n}^{\frac{\sigma(q-2)}{q}}\left\|V_{n}\right\|_{L^{q}}=e^{-2 a_{n-1}}\left(1+16 \lambda_{n}^{4} a_{n}^{-4}\right)^{-\frac{N(q-2)}{4 q}} \lambda_{n}^{\frac{\sigma(q-2)}{q}}\left(\frac{\pi}{q}\right)^{\frac{N}{2 q}} \underset{n \rightarrow \infty}{\longrightarrow} c .
$$

We finally estimate $W_{n}$. It follows from (2.2) that

$$
\left\|e^{i \lambda_{n}^{2} \Delta}\left[\theta\left(\cdot / a_{j}\right)\right]\right\|_{L^{q}} \leq C a_{j}^{\frac{N}{q}} .
$$

Therefore,

$$
a_{j}^{-\frac{N}{r}} e^{-2 a_{j-1}}\left\|e^{i \lambda_{n}^{2} \Delta}\left[\theta\left(\cdot / a_{j}\right)\right]\right\|_{L^{q}} \leq C e^{-2 a_{j-1}},
$$

so that

$$
\left\|W_{n}\right\|_{L^{q}} \leq C \sum_{j=n+1}^{\infty} e^{-2 a_{j-1}} \leq C \sum_{j=n}^{\infty} e^{-2 a_{j}} \leq C e^{-a_{n}},
$$

by (2.3). We deduce from the above estimate, (2.11) and (2.3) that

$$
\lambda_{n}^{\frac{\sigma(q-2)}{q}}\left\|W_{n}\right\|_{L^{q}} \leq C \lambda_{n}^{\frac{\sigma(q-2)}{q}} e^{-a_{n}} \underset{n \rightarrow \infty}{\longrightarrow} 0 .
$$

Now setting $t_{n}=\lambda_{n}^{2}$, we find that the estimate (1.7) follows from (2.12), (2.13), (2.14) and (2.15). This completes the proof, since $q, \sigma$ and $c$ are arbitrary.

Proof of Theorem 1.5. We use the initial value constructed in Theorem 1.1 and a perturbation argument. We set

$$
\beta=\frac{4-(N-2) \alpha}{2 \alpha(\alpha+2)}, \quad \mu=\frac{N \alpha}{4(\alpha+2)} .
$$

It easily follows from (2.16) and (1.9) that

$$
\begin{gathered}
0<\beta<\mu<\frac{1}{2}, \\
\alpha \beta+2 \mu=1 .
\end{gathered}
$$

Let $\psi$ be as given by Theorem 1.1 with $r=2$. It follows that $\psi \in H^{1}\left(\mathbb{R}^{N}\right)$,

$$
\sup _{t \geq 0}(1+t)^{\frac{N(q-2)}{4 q}}\left\|e^{i t \Delta} \psi\right\|_{L^{q}}<\infty,
$$

for all $2<q \leq \infty$, and that for all

$$
0<\eta<\frac{N(q-2)}{4 q},
$$


and all $0 \leq c<\infty$, there exists a sequence $t_{n} \rightarrow \infty$ such that

$$
t_{n}^{\frac{N(q-2)}{4 q}+\eta}\left\|e^{i t_{n} \Delta} \psi\right\|_{L^{q}} \underset{n \rightarrow \infty}{\longrightarrow} c .
$$

We now let

$$
\varphi=\varepsilon \psi,
$$

where $\varepsilon>0$ is to be chosen sufficiently small. Since $\psi \in H^{1}\left(\mathbb{R}^{N}\right)$, it follows that if $\varepsilon$ is sufficiently small, then the solution of (1.8) is global and bounded in $H^{1}\left(\mathbb{R}^{N}\right)$. This proves part (i). Moreover, we deduce from (2.19) that $(1+t)^{\mu}\left\|e^{i t \Delta} \varphi\right\|_{L^{\alpha+2}} \leq$ $\varepsilon C$. Since $\beta(\alpha+1)+(\mu-\beta)<1$ by (2.18), we may apply Theorem 2.1 in [6] (with $\delta=\mu-\beta$ ), and we obtain $t^{\mu}\|u(t)\|_{L^{\alpha+2}} \leq C$ by choosing $\varepsilon>0$ possibly smaller. (Note that the solution $u$ and the solution given by Theorem 2.1 in $[6$ coincide. See Proposition 2.3 (f) in 6].) Since $u \in L^{\infty}\left((0, \infty), H^{1}\left(\mathbb{R}^{N}\right)\right)$ and $H^{1}\left(\mathbb{R}^{N}\right) \hookrightarrow$ $L^{\alpha+2}\left(\mathbb{R}^{N}\right)$, we conclude that

$$
\sup _{t \geq 0}(1+t)^{\mu}\|u(t)\|_{L^{\alpha+2}}<\infty .
$$

We next observe that

$$
u(t)-e^{i t \Delta} \varphi=-i \lambda \int_{0}^{t} e^{i(t-s) \Delta}|u(s)|^{\alpha} u(s) d s .
$$

Using the estimate $\left\|e^{i \tau \Delta} w\right\|_{L^{\alpha+2}} \leq C \tau^{-2 \mu}\|w\|_{L^{\frac{\alpha+2}{\alpha+1}}}$, we deduce from (2.23)-(2.24) that

$$
\begin{aligned}
\left\|u(t)-e^{i t \Delta} \varphi\right\|_{L^{\alpha+2}} & \leq C \int_{0}^{t}(t-s)^{-2 \mu}\|u(s)\|_{L^{\alpha+2}}^{\alpha+1} \\
& \leq C \int_{0}^{t}(t-s)^{-2 \mu}(1+s)^{-(\alpha+1) \mu} .
\end{aligned}
$$

Note that $(\alpha+1) \mu<1$ if $N \geq 6$, but $(\alpha+1) \mu>1$ if $N \leq 5$ and $\alpha$ is sufficiently large. Thus we need to consider the three cases $(\alpha+1) \mu<1,(\alpha+1) \mu=1$ and $(\alpha+1) \mu>1$. It is not difficult to show that for $t$ large,

$$
\int_{0}^{t}(t-s)^{-2 \mu}(1+s)^{-(\alpha+1) \mu} \leq \begin{cases}C t^{-\mu-\alpha(\mu-\beta)} & \text { if }(\alpha+1) \mu<1, \\ C t^{-2 \mu} \log t & \text { if }(\alpha+1) \mu=1, \\ C t^{-2 \mu} & \text { if }(\alpha+1) \mu>1 .\end{cases}
$$

It follows from (2.26) that in all three cases there exists $0<\delta \leq \mu$ such that

$$
\int_{0}^{t}(t-s)^{-2 \mu}(1+s)^{-(\alpha+1) \mu} \leq C t^{-\mu-\delta} .
$$

By applying (2.25) and (2.27), we conclude that

$$
t^{\mu}\left\|u(t)-e^{i t \Delta} \varphi\right\|_{L^{\alpha+2}} \leq C t^{-\delta} .
$$

We now observe that both $u(t)$ and $e^{i t \Delta} \varphi$ are bounded in $H^{1}\left(\mathbb{R}^{N}\right)$, uniformly in $t \geq 0$. By applying Hölder's inequality

$$
\|w\|_{L^{q}} \leq\|w\|_{L^{\alpha+2}}^{\frac{(\alpha+2)(q-2)}{\alpha q}}\|w\|_{L^{2}}^{\frac{2(\alpha+2)-2 q}{\alpha q}}
$$

for $2 \leq q \leq \alpha+2$, we deduce from (2.28) that

$$
t^{\frac{N(q-2)}{4 q}}\left\|u(t)-e^{i t \Delta} \varphi\right\|_{L^{q}} \leq C t^{-\frac{\delta(\alpha+2)(q-2)}{\alpha q}},
$$


for all $2 \leq q \leq \alpha+2$. Next, by applying Gagliardo-Nirenberg's inequality

$$
\|w\|_{L^{q}} \leq C\|\nabla w\|_{L^{2}}^{\frac{2 N[q-(\alpha+2)]}{q[(-(N-2) \alpha]}}\|w\|_{L^{\alpha+2}}^{\frac{(\alpha+2)[4-(N-2)(q-2)]}{q[(N-2) \alpha]}}
$$

for $\alpha+2 \leq q<2 N /(N-2)^{+}$, we deduce from (2.28) that

$$
t^{\frac{N(q-2)}{4 q}}\left\|u(t)-e^{i t \Delta} \varphi\right\|_{L^{q}} \leq C t^{-\frac{\delta(\alpha+2)[2 N-(N-2) q]-N[q-(\alpha+2)]}{q[4-(N-2) \alpha]}},
$$

for $\alpha+2 \leq q<2 N /(N-2)^{+}$. We define $\alpha+2<q_{0}<2 N /(N-2)^{+}$by

$$
\delta(\alpha+2)\left[2 N-(N-2) q_{0}\right]=N\left[q_{0}-(\alpha+2)\right] .
$$

For all $\alpha+2<q<q_{0}$ we define $r(q)>0$ by

$$
r(q)= \begin{cases}\frac{\delta(\alpha+2)(q-2)}{\alpha q} & \text { if } 2<q \leq \alpha+2, \\ \frac{\delta(\alpha+2)[2 N-(N-2) q]-N[q-(\alpha+2)]}{q[4-(N-2) \alpha]} & \text { if } \alpha+2 \leq q<q_{0},\end{cases}
$$

and we set

$$
\delta(q)=\min \left\{r(q), \frac{N(q-2)}{4 q}\right\} .
$$

We deduce from (2.29), (2.30) and (2.33) that

$$
t^{\frac{N(q-2)}{4 q}}\left\|u(t)-e^{i t \Delta} \varphi\right\|_{L^{q}} \leq C t^{-\delta(q)},
$$

for all $2<q<q_{0}$. Estimate (1.11) now follows from (2.19) and (2.34), while (1.12) follows from (2.21) and (2.34). This shows part (ii) and completes the proof.

\section{ACKNOWLEDGEMENTS}

The authors thank the referee for constructive remarks concerning the exposition of this article. The first two authors thank Professor Fang Daoyuan for fruitful discussions.

\section{REFERENCES}

[1] Beckner, W. Inequalities in Fourier analysis, Ann. of Math. (2) 102 (1975), no. 1, 159-182. MR0385456 (52:6317)

[2] Bégout, P. Maximum decay rate for finite-energy solutions of nonlinear Schrödinger equations, Differential Integral Equations 17 (2004), 1411-1422. MR2100034(2005i:35241)

[3] Cazenave, T. Semilinear Schrödinger equations, Courant Lecture Notes in Mathematics, 10. New York University, Courant Institute of Mathematical Sciences, New York; American Mathematical Society, Providence, RI, 2003. MR2002047 (2004j:35266)

[4] Cazenave, T., Dickstein, F. and Weissler, F. B. A solution of the heat equation with a continuum of decay rates, in Elliptic and parabolic problems: A special tribute to the work of Haïm Brezis, Progress in Nonlinear Differential Equations and Their Applications, 63. Birkhäuser-Verlag, Basel, 2005, 135-138. MR2176707 (2006e:35144)

[5] Cazenave, T. and Weissler, F. B. Rapidly decaying solutions of the nonlinear Schrödinger equation, Comm. Math. Phys. 147 (1992), 75-100. MR1171761 (93d:35150)

[6] Cazenave, T. and Weissler, F. B. Asymptotically self-similar global solutions of the nonlinear Schrödinger and heat equations, Math. Z. 228 (1998), 83-120. MR.1617975 (99d:35149)

[7] Cazenave, T. and Weissler, F. B. Scattering theory and self-similar solutions for the nonlinear Schrödinger equation, SIAM J. Math. Anal. 31 (2000), 625-650. MR1745480 (2001h:35169)

[8] Cazenave, T. and Weissler, F. B. Spatial decay and time-asymptotic profiles for solutions of Schrödinger equations, Indiana Univ. Math. J. 55, no. 1 (2006), 75-118. MR 2207548 (2007d:35224)

[9] Fang, D., Xie, J. and Cazenave, T. Multiscale asymptotic behavior of the Schrödinger equation, in preparation.

[10] Ginibre, J. and Velo, G. On a class of nonlinear Schrödinger equations. I. The Cauchy problem, general case, J. Funct. Anal. 32, no. 1 (1979), 1-32. MR533218 (82c:35057) 
[11] Kato, T. An $L^{q, r}$-theory for nonlinear Schrödinger equations, in Spectral and scattering theory and applications, Advanced Studies in Pure Mathematics, 23, Math. Soc. Japan, Tokyo, 1994, 223-238. MR1275405 (95i:35276)

[12] Strauss, W.A. Nonlinear scattering theory, in Scattering theory in mathematical physics, J. A. Lavita and J.-P. Marchand (eds.), Reidel, 1974, 53-78.

Department of Mathematics, Zhejiang University, Hangzhou, Zhejiang, 310058, PeoPle's Republic of ChinA

E-mail address: sword711@gmail.com

Department of Mathematics, Zhejiang University, Hangzhou, Zhejiang, 310058, PeoPLE'S REPUBLiC OF CHINA

E-mail address: linzi0116@gmail.com

Université Pierre et Marie Curie \& CNRS, Laboratoire Jacques-Louis Lions, B.C. 187, 4 Place Jussieu, 75252 Paris Cedex 05, France

E-mail address: thierry.cazenave@upmc.fr 